\title{
Modelos y teorías computacionales de la memoria humana: un estado de la cuestión y análisis crítico
}

\section{Computational Models and Theories of Human Memory: a State of the Situation and Critical Analysis}

\author{
Renato Garita Figueiredo \\ Programa en Fundamentos de Educación a Distancia \\ Universidad Estatal a Distancia \\ Colaboratorio Nacional de Computación Avanzada \\ Centro Nacional de Alta Tecnología \\ San José, Costa Rica \\ rgarita@uned.ac.cr / renatogarita@cenat.ac.cr / renatogarita@gmail.com
}

Recibido 26-08-2010 • Aceptado 21-09-2010 • Corregido 09-10-2010

\begin{abstract}
Resumen: Las funciones de la memoria están intimamente ligadas a todos los procesos cognitivos humanos. El estudio de la memoria humana está sumido en un sinnúmero de teorías y modelos de toda indole, por lo que es necesario aclarar el panorama de estudio para crear mejores aplicaciones en educación $y$ en otras áreas relacionadas. Este artículo sobre modelos y teorías computacionales de la memoria es parte de una línea de investigación en memoria humana que busca crear un estado de la cuestión sobre el peso de los estudios actuales en el área. Se realiza una revisión crítica de 37 artículos sobre computación y memoria utilizando un instrumento de análisis que se ha denominado "tamiz". Se identifican los aspectos más relevantes encontrados en estos artículos y se concluye con unas observaciones generales sobre lo encontrado. Los resultados muestran que hay una gran dependencia a la utilización de la metáfora computacional de la mente para explicar los procesos cognitivos de los seres humanos.
\end{abstract}

Palabras clave: Memoria humana, computación, metáfora computacional, inteligencia artificial.

\section{Introducción}

Los resultados de los estudios sobre memoria humana influyen considerablemente en muchas disciplinas y aplicaciones donde es importante comprender los procesos cognitivos superiores de los seres humanos. Este es un tema de estudio que ha causado una proliferación de investigaciones y aplicaciones en diversidad de disciplinas, lo cual ha dificultado que haya una visión clara del panorama que permita hacer un mejor análisis y crítica a la hora de aplicar los resultados.

Este artículo sobre computación y memoria es parte de una línea de investigación de la memoria humana planteada en el marco del Programa en Fundamentos de la Educación a Distancia (PROIFED) de la Universidad Estatal a Distancia (UNED) y, como parte de sus objetivos, se pretende plantear una serie 


\begin{abstract}
Memory tasks are closely linked to all our cognitive processes. The study of human memory is immersed in countless theories and models of every sort, so it is necessary to clear the outlook of study to create better applications in education and other related areas. This article is about computational models of human memory, and is part of a line of research on human memory, which goal is to demonstrate the importance of the most recent studies in the area. With this investigation, we made a critical review of 37 papers about subjects of computation and memory, using an analysis instrument which we called "tamiz." We identified the most relevant aspects found in these papers and concluded with general observations about what we found. The results show a large reliance on the mind-computer metaphor to explain the cognitive processes of human beings.
\end{abstract}

Key words: Human memory, computation, computational metaphor, artificial intelligence. de proyectos que analicen, propongan e implementen modelos y aplicaciones sobre memoria humana en áreas relacionadas a la educación.

El primer proyecto de esta línea se denomina "La memoria: Análisis y evaluación crítica de las teorías, modelos $y$ enfoques actuales"; en este, se busca hacer una revisión de propuestas sobre memoria humana que abarque el peso de la investigación en la actualidad y analice críticamente cuáles son sus fundamentos teóricos y características. En él se realiza un estado de la cuestión y un análisis crítico sobre modelos de la memoria en los ámbitos de psicología, neurociencia, computación y estudios del lenguaje. El presente artículo tiene como objetivo mostrar los resultados de un análisis crítico del estado de la cuestión en el área de estudio de la computación y la memoria donde mucha de la investigación sobre memoria humana se da desde la perspectiva de la inteligencia artificial (IA) y con la utilización de fundamentos sobre la visión de una mente humana que funciona con mecanismos computacionales.

En primerainstancia, los antecedentes del estudio de la cognición desde el enfoque computacional ayudarán a poner en perspectiva el panorama de estudio. Luego se desglosará lo encontrado con un documento de revisión, que se ha denominado "tamiz"1, en el cual se revisa una serie de trabajos que involucran a la memoria humana y a la computación. Algunos de estos artículos tratan abiertamente sobre visiones de la memoria como componente(s) de un sistema computacional. Otros tratan sobre la creación de aplicaciones computacionales que mejoren la utilización de la memoria. Por otra parte, otros se limitan a describir formas de simular tareas específicas de la memoria. Seguidamente se hará una discusión breve de lo encontrado con la revisión, lo cual sentará la base para proponer una serie de consideraciones que deben darse en los estudios sobre memoria.

No es objetivo de este artículo realizar una explicación exhaustiva de los términos 
IA y metáfora computacional debido a que son términos muy complejos. Se considera prudente, eso sí, hacer una breve aclaración sobre estos temas ya que serán de uso recurrente en el cuerpo de este trabajo.

\section{Inteligencia artificial}

Cuando se habla de computación en el ámbito de la cognición se refiere más a una visión general y teórica sobre cómo funcionan y cómo se estructuran ciertos mecanismos mentales, y no tanto a implementaciones en la computadora digital, que es como se entiende a la IA principalmente. A pesar de esto, dado que la teoría detrás de estos supuestos cognitivos coincide con la teoría detrás de la computadora digital, la implementación de los modelos y aplicaciones en máquinas computacionales es muchas veces deseable y recomendada y es por eso que la IA se considera un tema fundamental para el desarrollo de esta visión de la mente computacional.

El término inteligencia artificial fue concebido por John McCarthy en 1955 como propuesta a los participantes de la conferencia de Dartmouth (McCarthy, Minsky, Rochester y Shannon, 1955) y se refería a la creación de máquinas inteligentes. Inteligencia en este sentido se entiende como la inteligencia humana, por lo que se esperaba que la máquina computacional pudiera mostrar inteligencia como la de los seres humanos ${ }^{2}$. Por algún tiempo los esfuerzos de la investigación en IA se fueron por este lado, y fue el Solucionador General de Problemas (GPS por sus siglas en inglés) de Alan Newell y Herbert Simon uno de los esfuerzos más famosos donde se simulaba la forma de razonar lógicamente de los seres humanos (Newell y Simon, 1959). Posteriormente, debido a presiones del mercado que exigían mejores resultados, el término empieza a utilizarse para denominar inteligencia computacional en un sentido más práctico donde no necesariamente es la inteligencia humana lo que se intenta replicar, sino que son máquinas que exhiben ciertos comportamientos que pueden ser considerados como inteligentes y que sirven para aplicaciones específicas. La IA más tradicional retomó fuerza brevemente para luego recaer en los años 80 envuelta en una serie de críticas que cuestionaban sus supuestos (McCurdock, 2004³; Russell y Norvig, 20094). Los interesados en el tema empezaron a denominar IA débil a todos los esfuerzos que no eran IA tradicional, por lo que la IA como herramienta puede considerarse IA débil. Por su lado, la IA tradicional pasó a denominarse IA fuerte. Algunos de los críticos de la IA fuerte, como John Searle, denominaban IA débil a todo aquel esfuerzo cuyo objetivo principal no fuera una reproducción (emulación) fiel de inteligencia humana en la máquina computacional. Dentro de lo que Searle considera como IA débil están los esfuerzos por simular o comprender la cognición humana utilizando a la máquina computacional siempre y cuando se tenga claro que lo que simula no es el fenómeno real (Searle, 1980).

En la actualidad, la inteligencia artificial es vista principalmente como una herramienta para aplicaciones en computación como Microsoft Office (Nordlander, 2001), tanto así que lo que originalmente pudo haberse considerado como IA hoy en día no es llamado por ese nombre, debido a que el concepto que se maneja de inteligencia no busca comparar directamente la inteligencia humana con la computadora. Hoy en día la mayoría de los esfuerzos en IA son comerciales y a manera de herramientas aunque haya algunos que todavía esperan reproducir verdadera inteligencia en computadoras y otros que la utilizan para prueba de hipótesis (Sun, 2009). Muchos de los esfuerzos comerciales implementan también algoritmos o estructuras representacionales que se basan en teorías sobre cómo funciona la mente humana, toda esta retroalimentación y diversificación que ha sufrido la IA ha provocado que no sea fácil 
una identificación clara entre IA fuerte y débil. Sin embargo, en aras de la claridad, cuando se habla de IA fuerte y débil en este artículo se deben considerar como lo fueron originalmente: la IA fuerte como una forma de reproducir inteligencia humana en la computadora y la débil como todo lo demás, es decir, IA que se utiliza ya sea para simulación de procesos cognitivos humanos pero que no intenta reproducir o representar verdadera cognición e IA del tipo de aplicaciones comerciales que buscan resolver un problema aplicando algoritmos que hacen que la máquina se comporte de manera aparentemente inteligente; así mismo, la combinación de ambas. La distinción puede volverse confusa en algunos casos ya que la diferencia entre fuerte y débil depende muchas veces de la intensión (no siempre explícita) del investigador y no tanto de la forma en que esté construida su aplicación.

\section{Metáfora computacional}

A pesar de la evolución del concepto de IA y los múltiples fracasos que rodearon a la disciplina, los supuestos de fondo que inspiraron el optimismo inicial se mantienen muy vigentes en el estudio de las capacidades mentales humanas. A partir de la mitad del siglo pasado, la computación es una disciplina que ha influido grandemente en la percepción de la cognición humana. Si bien muchos de los principios de este enfoque no tienen fundamento como se sabe actualmente, se sigue dependiendo, casi que de forma tácita, en los supuestos que explican los procesos mentales humanos en términos computacionales, y muchas de las disciplinas que estudian la memoria o utilizan los supuestos ligados a la memoria como parte de otra línea de investigación los utilizan. Debido a esto, se espera que gran parte del estudio que involucra a la computación y la memoria muestre este tipo de sesgos.

La teoría dela metáfora computacional de la mente es propuesta por Hilary Putnam en 1961 y desarrollada por Jerry Fodor en las décadas siguientes (Horst, 2005). Una de sus bases es la manipulación formal de símbolos la cual proviene de la matemática, donde se utilizó para fundamentar el razonamiento matemático en axiomas y reglas. La formalización matemática acarrea la relación semántica entre símbolos de un sistema, por lo que resultaba muy práctico para describir sistemas matemáticos como la geometría y la aritmética. Sin embargo, Goedel probó que un sistema formal consistente no es suficiente para dar cuenta de todos los enunciados verdaderos en aritmética con su teorema de incompletitud (Dawson, 2006). Por su parte, Alan Turing describió una máquina que podía computar razonamientos similares a los humanos con cualquier operación sintácticamente manipulable (Turing, 1950). De esta forma, se relaciona la computación a la formalización de estructuras simbólicas y surge la idea de que el razonamiento humano es de esta naturaleza. ${ }^{5}$

Debido a esta relación estrecha entre computación y formalismo, la IA emerge como el área de aplicación predilecta para estas teorías y de ahí que se desarrollen en un periodo similar. Por su parte, las estructuras de representación del conocimiento y de módulos con funciones específicas que se encuentran en la IA retroalimentan disciplinas que habían tomado interés en la metáfora computacional de la mente, como la psicología y la ciencia cognitiva, la cual se convierte en la disciplina que adopta los supuestos de la metáfora computacional como parte de sus bases teóricas (Von Eckardt, 1993).

De ahí en adelante las funciones cognitivas pasan a verse como mecanismos computacionales o equivalentes a mecanismos computacionales. La memoria es una de las funciones cognitivas que se ve influenciada por la manera de representar el conocimiento en módulos con estructuras simbólicas formales y en redes conexionistas. Mucha de esta visión de la cognición se mantiene vigente en estudios contemporáneos. 
Habiendo aclarado los términos de IA y metáfora computacional de forma breve, la siguiente sección tratará directamente sobre la revisión crítica del estado de la cuestión.

\section{Metodología y resultados}

Con el avance de otras disciplinas que estudian la cognición, como la neurociencia, el tema de la computación y la IA como un marco de estudio de la cognición ha estado relegado más que todo a un segundo plano donde cumple un papel de instrumento modelador y simulador complementario. Es evidente, sin embargo, que los principios de la mente computacional siguen siendo relevantes en muchas áreas ajenas a la computación y en muchos casos estos principios son utilizados de forma tácita. Debido a la pérdida de protagonismo de la IA en los estudios de cognición, la investigación en el campo donde el modelo computacional es el enfoque principal es escasa. Un análisis de la utilización de la metáfora computacional en otras disciplinas como la neurociencia, la psicología y el estudio del lenguaje se puede también encontrar en los estados de la cuestión complementarios que son parte del proyecto "La memoria: Análisis y evaluación crítica de las teorías, modelos y enfoques actuales".

Dos de los enfoques que tratan el tema de la memoria en el área de la computación lo hacen de forma más directa como lo son las arquitecturas cognitivas y los modelos computacionales de tipos o funciones de memoria específicas, el tercer enfoque identificado trata el tema de la memoria de forma más indirecta con el tema de aplicaciones de interfaz humano-computador y memorias prostéticas, cuyo principal objetivo es, en la mayoría de los casos, el diseño e implementación de una aplicación. Se realizó una revisión superficial de más de 100 artículos sobre temas relacionados; de esta revisión inicial se escogieron $37^{6}$ que fueron analizados más detalladamente con el tamiz y que se cree son una buena representación del peso del estudio actual sobre memoria desde una perspectiva computacional. La selección de los 37 artículos se hizo utilizando criterios subjetivos concernientes a qué tanto se hace referencia a la computación como el enfoque central del trabajo; asimismo, se buscaron principalmente artículos recientes pero, en algunos casos, se complementó con artículos de más antigüedad para evaluar el cambio de visión que ha sufrido el área. Se dejaron por fuera varias propuestas que utilizan la computación como instrumento modelador o simulador pero cuyo énfasis principal es en los resultados de estudios en otras disciplinas como la neurociencia y la psicología; esta decisión fue tomada ya que el proyecto del que es parte este artículo contempla también la revisión por otros investigadores de bibliografía en estas áreas.

\section{Clasificación}

La revisión bibliográfica dejó como resultado una clasificación que se hizo con base en el objetivo práctico de los estudios. No se realizó una clasificación de los artículos en teorías específicas sobre tipos y funciones de memoria, ya que la mayoría de propuestas coinciden en una visión general de la memoria como sistema computacional, por lo que los supuestos de fondo no varían considerablemente. En los casos en que hubo una desviación importante en las concepciones de fondo se mencionará y se desarrollará en la sección de la discusión.

Las tres áreas de estudio identificadas en las propuestas fueron: aplicaciones de interfaz humano-computador y de memorias prostéticas, arquitecturas cognitivas y modelos de funciones o tipos específicos de memoria.

\section{Sistemas de interfaz humano-computador y de memorias prostéticas}

Las propuestas que se clasifican aquí utilizan los resultados de investigaciones o supuestos teóricos sobre memoria humana 
para proponer aplicaciones que asistan a usuarios a mejorar su memoria en tareas o áreas de trabajo específicas (Kallinen, Laarni, Ravaja y Saari, 2004; Sas y Dix, 2006; Aizenbud-Reshef, Belinsky, Jacovi, Laufer y Soroka, 2008; Commaford, 2006; Dekel, 2008; Elsweiler, 2007; Hewett, 2005; Huggett, Hoos y Rensink, 2007; Huguenard, Lerch, Junker, Patz y Kass, 1997; Kawamura, Fukuhara, Takeda, Kono y Kidode, 2006; Schweer y Hinze, 2007; Steyvers, Griffiths y Dennis 2006; van den Hoben y Eggen, 2007; Vemuri, Schmandt y Bender, 2006; West, Quigley, A. y Kay, 2007).

Lo interesante de estas aplicaciones es que muchas de las estructuras de datos que en ellas se proponen hacen referencia a estructuras de representación mental. Las que tratan sobre interfaces humanocomputador, por su parte, hacen referencia principalmente a características de la memoria o funciones y tipos de memoria que pueden ser beneficiados debido al uso de los diseños de interfaz.

El fuerte sesgo de la visión modular y representacional de la mente que acompaña a la teoría de la metáfora computacional se encuentra presente en muchas de las propuestas. Solamente en algunas de ellas se considera importante un componente emocional que va de la mano con el rendimiento de la memoria (Kallinen et ál., 2004) y en otros se toca el tema de la interacción social como un aspecto que requiere un análisis más a fondo (Sas et ál., 2006). En los casos analizados no se profundiza mucho en la fundamentación teórica debido a que los planteamientos de las propuestas tienen como objetivo primordial diseñar aplicaciones comerciales.

\section{Arquitecturas cognitivas}

El segundo tipo de propuestas que se identificaron fueron las que describen arquitecturas cognitivas. Estas propuestas buscan describir una arquitectura cognitiva computacional que represente fielmente los elementos básicos de la cognición humana sobre los cuales se puedan definir modelos de todo tipo (Anderson, 1996; Izquierdo, 2008; Marinier y Laird, 2004; Sun, 2004; Van Gelder, 1998; Lehman, Laird y Rosenbloom, 2006; Schoelles, Neth, Myers y Gray, 2006). De entre los tres tipos de propuestas que se revisaron, las aplicaciones de este ámbito son las que más se apegan a la concepción de IA fuerte, ya que definen una teoría unificada de la cognición. Aún así, en algunos casos puede que se utilice como una herramienta de simulación, lo que haría que se clasifique dentro de IA débil.

La forma en que se justifican las propuestas es mediante la hipótesis de que la cognición es principalmente resultado de mecanismos computacionales básicos de manipulación de estructuras simbólicas. Las propuestas conexionistas buscan describir arquitecturas cognitivas basadas en mecanismos sub-simbólicos, pero en la práctica siempre es necesario un nivel simbólico para tareas de más alto nivel ${ }^{7}$.

Las propuestas revisadas generalmente combinan estructuras representativas simbólicas con parámetros de activación sub-simbólicos, lo cual hace que sean acercamientos híbridos. Los experimentos realizados con estas arquitecturas buscan principalmente reproducir el comportamiento humano en tareas de experimentos psicológicos de laboratorio, como la recuperación de hileras de palabras de la memoria; por ejemplo, véase Anderson, Bothell, Lebiere y Matessa (1998).

Existen esfuerzos por reproducir comportamientos complejos pero las limitaciones de una arquitectura basada en subsistemas relativamente aislados provocan serios problemas para explicar diferencias considerables entre el comportamiento de un ser humano y del modelo computacional, y las propuestas complejas no son fáciles de implementar (Schoelles et ál., 2006). El nivel de detalle en estos modelos es generalmente muy 
profundo, por lo que se espera que se pueda reproducir el comportamiento incluso hasta en los niveles de tiempo de respuesta. Estas propuestas siguen la sugerencia de Allan Newell (Newell, 1990), quien dice que para comprender la cognición de forma adecuada se necesitan teorías unificadas de la cognición, por lo que la memoria es solo un sub-sistema de estas arquitecturas y se ve en relación con los demás sub-sistemas cognitivos.

Las principales propuestas revisadas son de la arquitectura cognitiva Adaptive Character of Thought (Act-R) de J. R. Anderson (Anderson, 1996; Anderson y Betz, 2002; Anderson et ál., 1998; Anderson, Budiu Reder, 2001;), por lo que se realizaron pruebas para experimentar con la complejidad de la arquitectura.

\section{Pruebas}

Una serie de pruebas se realizaron con modelos en la arquitectura cognitiva Act- $R$ en un esfuerzo por comprender el nivel de complejidad requerido para simular el comportamiento humano adecuado. Las pruebas realizadas fueron tomadas de los tutoriales en el sitio de internet de Act-R Research Group (s. f.), realizadas con la asistencia del Centro Nacional de Alta Tecnología (CeNAT) y son:

- $\quad$ Prueba de Sterling: consiste en presentar una matriz de letras al individuo y que éste intente recordar las letras de una de las filas de la matriz. Es un modelo simbólico.

- Prueba de pares: consiste en presentar al individuo pares de palabras y números para que luego los recuerde. Es un modelo híbrido.

- Prueba de Zbrodoff: consiste en pedirle al individuo que juzgue problemas aritméticos con el alfabeto. Por ejemplo, se presenta la ecuación $\mathrm{A}+2=\mathrm{C}$ y el individuo debe responder sí o no según considere que se justifique la ecuación a partir del valor posicional de las letras (ej. $A=1, B=2$, etc). Es un modelo híbrido.

- Prueba de propagación: consiste en presentar al individuo una serie de oraciones con personajes y localizaciones que se repiten. Al individuo se le presentan luego otras oraciones y debe juzgar si se encontraban en la lista original. Es un modelo híbrido.

Todas las pruebas presentan diferentes niveles de detalle en cuanto a los parámetros sub-simbólicos que es necesario definir y calibrar. El nivel de detalle y prueba de error para incluso las pruebas más simples exige un esfuerzo considerable en el tiempo y en la calibración de cada modelo por separado por parte del investigador.

\section{Modelos de tipos de memoria específicos}

La tercera clasificación trata sobre modelos de tipos de memoria o funciones de la memoria específicas (Baddeley, 1986; Burgess, 2000; Burgess y Hitch, 2005; Byrne, 1996; Chartier, Renaud y Boukadoum, 2008; Collins, 1973; Denhière y Lemaire, 2004; Foltz, 1991; Howard, 2009; Leake, 1994; Norman, Detre y Polyn, 2008; O'Reilly, Braver y Cohen, 1999; Rizzuto, 2002; Rogers, 2008; Shastri, 2001). En este tipo de modelo se aisla alguna característica o función de la memoria para ser estudiada aparte de los otros procesos cognitivos. Existen modelos que describen la memoria de trabajo, memoria a corto o largo plazo, memoria procedimental o declarativa, memoria semántica o episódica, etc. Las aplicaciones de muchos de estos modelos pueden considerarse como IA débil, como es concebida por Searle, ya que se utilizan como simulaciones de mecanismos muy específicos de la memoria.

Dentro de este tipo de propuestas los modelos conexionistas son más utilizados que con las propuestas de 
arquitecturas cognitivas, ya que pueden crearse para modelar mecanismos aislados y muy específicos sin necesidad de las representaciones simbólicas más tradicionales. Asimismo, existen teorías y modelos puramente simbólicos que intentan explicar las diferencias entre los tipos de memoria y en cómo interactúan.

Dentro de este tipo de propuestas la metáfora computacional está igualmente presente en casi todas los estudios aunque existan también propuestas con planteamientos diferentes a partir de modelos dinámicos (Chartier et ál., 2008).

\section{Resultados estadísticos}

Esta sección muestra un desglose estadístico y sintético de lo encontrado con respecto a la revisión bibliográfica que se realizó, esto con el fin de elucidar cuáles son las características principales de las propuestas revisadas.

En total se revisaron 37 propuestas con temáticas variadas dentro del área que estudia la memoria desde la perspectiva computacional.

De las ramas descritas previamente, 15 de las propuestas son de la rama de aplicaciones de memoria prostética y de interfaz humano-computador, 7 son de arquitecturas cognitivas y 15 son sobre modelos o teorías de funciones o mecanismos específicos de la memoria humana.

\section{Origen}

Las 37 propuestas tienen su origen en la informática, computación y/o IA. De estas, 20 hacen énfasis en la psicología como una de las fuentes de sus teorías, 5 en la neurociencia y 11 en las ciencias cognitivas. A pesar de que prácticamente todas las propuestas tengan fundamentos evidentes en la teoría de la metáfora computacional de la mente, la cual proviene de la ciencia cognitiva, solamente algunas (11) hacen referencia explícita del área como influencia principal.
Cuadro 1

Origen de las propuestas

\begin{tabular}{|l|c|}
\hline Origen & Total \\
\hline Informática y/o IA & $37(100 \%)$ \\
\hline Psicología & $20(54 \%)$ \\
\hline Neurociencia & $5(13,5 \%)$ \\
\hline Ciencia Cognitiva & $12(32,4 \%)$ \\
\hline
\end{tabular}

\section{Autodefinición}

Delas 37 propuestas 12 se autodefinen como hipótesis, 7 como teoría, 19 como modelo, 13 como sistemas de informática y 1 era la propuesta de un seminario. Muchas propuestas pueden ser clasificadas en más de una categoría y otras no hacen una definición explícita de lo que desarrollan, por lo que la clasificación se basa en criterios subjetivos basados en la introducción y justificación de las propuestas.

\section{Cuadro 2}

Autodefinición de las propuestas

\begin{tabular}{|l|r|}
\hline \multicolumn{1}{|c|}{ Autodefinición } & Total \\
\hline Hipótesis & $12(32,4 \%)$ \\
\hline Teoría & $7(18,9 \%)$ \\
\hline Modelo & $19(51,3 \%)$ \\
\hline Sistema de informática & $13(35,1 \%)$ \\
\hline Seminario & $1(2,7 \%)$ \\
\hline
\end{tabular}

\section{Definición luego del tamiz}

Luego del tamiz las propuestas se definieron según los objetivos o resultados obtenidos. Con respecto a esto, se identificaron 13 propuestas que se pueden considerar hipótesis, 9 teorías, 19 modelos, 14 aplicaciones de informática y un seminario. Igualmente se utilizó un método subjetivo para evaluar la naturaleza de las propuestas. La mayoría son coherentes en sus objetivos y los resultados que obtienen. 
Cuadro 3

Definición de las propuestas luego de someterlo al tamiz

\begin{tabular}{|l|r|}
\hline Definición luego del tamiz & Total \\
\hline Hipótesis & $13(35,1 \%)$ \\
\hline Teoría & $9(24,3 \%)$ \\
\hline Modelo & $19(51,3 \%)$ \\
\hline Sistema de informática & $14(37,8 \%)$ \\
\hline Seminario & $1(2,7 \%)$ \\
\hline
\end{tabular}

\section{Nivel de la propuesta}

La mayoría de las propuestas son descriptivas (33) y explicativas (25), hay pocas propuestas que proponen una teoría (9) y que realizan un trabajo de exploración profundo del área (6). Esto evidencia que los autores no se preocupan por cuestionar los fundamentos de fondo y se limitan a adoptar teorías existentes para desarrollar aplicaciones o modelos propios.

\section{Cuadro 4}

Nivel de la propuesta

\begin{tabular}{|l|c|}
\hline Nivel de la propuesta & Total \\
\hline Descriptivas & $33(88,2 \%)$ \\
\hline Explicativas & $25(67,6 \%)$ \\
\hline Teórica-X & $9(24,3 \%)$ \\
\hline Exploratorias & $6(16,2 \%)$ \\
\hline
\end{tabular}

\section{Alcance}

La mayoría de las propuestas se pueden considerar de corto (13) o de mediano (19) alcance en la investigación de memoria humana. Las que pueden considerarse como de mayor influencia son la minoría (3). Aquí de nuevo se evidencia que existe un consenso sobre lo que son los fundamentos computacionales de la cognición y de la memoria humana, pocas propuestas se alejan de estos supuestos para proponer algo relevante y nuevo en el área.
Cuadro 5

Alcance de la propuesta

\begin{tabular}{|l|c|}
\hline \multicolumn{1}{|c|}{ Alcance } & Total \\
\hline Corto & $13(35,1 \%)$ \\
\hline Mediano & $19(51,3 \%)$ \\
\hline Alto & $3(8,1 \%)$ \\
\hline Sin resultados concretos & $2(5,4 \%)$ \\
\hline
\end{tabular}

La propuesta categoriza o axiomatiza sus resultados

Se pudieron identificar 14 propuestas que axiomatizan los resultados mientras que se identificaron 12 que los categorizan. Esto, en combinación con el nivel de la propuesta, evidencia que existe una cantidad considerable de propuestas que se limitan a dar resultados confirmando modelos existentes (14). Otras propuestas (12) axiomatizan resultados que muchas veces añaden o refinan teorías ya existentes de forma superficial. Existen muy pocos estudios cuyas propuestas resalten axiomas que revolucionen el campo o aporten algo verdaderamente importante. En varias de las propuestas (11) no se dan resultados concretos que se puedan clasificar.

Cuadro 6

Categorización o axiomatización de los resultados

\begin{tabular}{|l|c|}
\hline Resultados & Total \\
\hline Categoriza & $14(37,8 \%)$ \\
\hline Axiomatiza & $12(32,4 \%)$ \\
\hline Sin resultados concretos & $11(29,7 \%)$ \\
\hline
\end{tabular}

\section{Metodología de las propuestas}

A pesar de haber propuestas de diferente índole, la mayoría utiliza una metodología similar a ésta, la cual es estándar en la creación de modelos computacionales generales: 
1. Creación de las reglas del modelo o aplicación computacional (ecuaciones y heurísticas) a partir de un modelo psicológico, neurocientífico o cognitivo; por ejemplo, la memoria semántica.

2. Alimentación del modelo con un cuerpo de datos recolectados en tareas llevadas a cabo por humanos. Este paso normalmente es obviado en las propuestas de aplicaciones de memorias prostéticas y de interfaz humano-computador, debido a que se toma el modelo como viene de la literatura para su implementación. En estos casos las pruebas, si existen, se realizan posteriormente a la creación de la aplicación.

3. Diseño y ejecución de experimentos computacionalesqueemulanosimulan las tareas realizadas por humanos 0 grupos de humanos en el caso de los modelos de tipos de memoria y de arquitecturas cognitivas. En el caso de las aplicaciones las pruebas se realizan midiendo las mejorías en el rendimiento de la memoria de las personas al utilizar la aplicación.

4. Análisis estadístico/experto de resultados.

5. Establecimiento de conclusiones.

No todas las propuestas vistas presentan las 5 etapas pero se puede generalizar que la gran mayoría sigue una metodología similar a la anterior. Por ejemplo, algunas de las propuestas se limitan a describir el modelo, sin embargo, no llegan a implementarlo ni mucho menos probarlo, pero se entiende que el objetivo es llevar a cabo este proceso eventualmente.

\section{Validez de las propuestas}

La validez del trabajo realizado en las propuestas se sustenta en sus bases teóricas de fondo. Estas bases provienen principalmente de propuestas teóricas psicológicas y cognitivas de la memoria humana como un mecanismo computacional. También se apoyan en ciertos casos en propuestas de la neurociencia que ligan comportamientos a patrones de activación neuronal.

Apegándose a la metodología, las propuestas que logran implementar un modelo o sistema basan su validez en la forma en que el modelo o sistema logra predecir y utilizar el comportamiento humano. Así, la simulación se sustenta en lograr resultados que se asimilen a la capacidad humana de hacer ciertas tareas de recuperación y retención de la memoria en los tiempos semejantes a los de un humano. Por ejemplo, las propuestas sobre sistemas de interfaz humano-computador y memorias prostéticas buscan que la implementación provoque las mejoras esperadas en mecanismos de la memoria humana para juzgar su validez.

A pesar de que todas las propuestas presentaban algún tipo de validación, ya sea teórica o empírica (donde la primera es la más común), casi ninguna presentaba una validación desde ambos frentes y que pueda considerarse completa para una propuesta científica.

\section{Fiabilidad de las propuestas}

El análisis de datos y los instrumentos estadísticos empleados son generalmente fiables y responden a los criterios tradicionales de minimización de incertidumbre y error aceptables en experimentos empíricos.

En los casos donde no se realizan experimentos empíricos los mismos criterios de validez que se utilizan para considerar que una propuesta teórica esté bien fundamentada le dan fiabilidad.

Mucha de la validación, sin embargo, requiere mantener supuestos teóricos de fondo muy fuertes, por lo que fuera de este marco no se pueden considerar como fiables sin un estudio crítico más profundo. 


\section{Plausibilidad de las propuestas}

La plausibilidad la sustentan los autores con la validez de los conceptos y teorías de fondo tomadas en cuenta en cada una de las propuestas y con los resultados de las pruebas empíricas. Esto no quiere decir que las propuestas sean buenos modelos de la memoria humana, ya que muchas se apoyan en la simulación computacional como un instrumento de verificación de procesos con bases en supuestos teóricos (de la metáfora computacional de la mente) que se encuentran todavía en debate.

En la aplicación de las teorías a explicar la memoria humana logran su objetivo parcialmente, ya que logran explicar mecanismos o funciones aisladas pero no existe propuesta que logre definir, explicar y probar un modelo de la memoria de forma completa e integral. Las implementaciones computacionales de los modelos se quedan cortos en resultados convincentes.

\section{Manejo del lenguaje, las emociones, la naturaleza social de la especie y las arquitecturas cognicionales en las propuestas}

La mayoría de las propuestas no toman en cuenta ninguna de estos aspectos en sus dimensiones más amplias. Se hacen menciones del lenguaje desde perspectivas sintácticas y semánticas (como contenido por la parte sintáctica) sin llegar a mucho más. Solamente algunas propuestas mencionan las emociones y naturaleza social de la especie como relevantes para los procesos de memoria humana (Kallinen et ál., 2004, Sas et ál., 2006, Sun, 2004, Marinier et ál., 2004).

\section{Concepto del ser humano}

Más que una noción de ser humano, las propuestas que forman parte de este estudio manejan una noción de memoria humana como un sistema computacional, con mecanismos de adquisición, asociación y recuperación de información. Es decir, el concepto de ser humano esta contenido en el cerebro o mente y aislado del medio.

Hay algunas propuestas que sí toman en cuenta la naturaleza social y emocional del ser humano y ven a la memoria como producto de algo más que la manipulación de estructuras formales simbólicas en sistemas aislados. Los autores de estas propuestas tienen la intuición de que se debe considerar una cognición humana social que involucra al cuerpo, entorno y emociones en diferente medida (Kallinen et ál., 2004, Sas et ál., 2006, Sun, 2004, Marinier et ál., 2004, Chartier et ál., 2008).

\section{Concepto de información y conocimiento}

El concepto de información de las propuestas es generalmente el de elementos simbólicos que forman estructuras o de elementos sub-simbólicos de activación que interactúan con estas estructuras o que asocian patrones de entrada con patrones de salida. El conocimiento se puede ver como estructuras de este tipo que se transforman con el tiempo y con nuevos insumos. Esto es influencia de la visión tradicional de la metáfora computacional donde el conocimiento es representado en forma de estructuras simbólicas formales.

Algunas propuestas sí consideran que el conocimiento se deriva de niveles más allá del cerebro, lo que está ligado a la visión particular del ser humano que se maneja en ellas como un ser social o emocional.

\section{Impacto de la propuesta}

Las propuestas no evidencian un impacto particular en la manera de ver el estudio de la memoria humana desde la perspectiva computacional. Esto se debe a que, en su mayor parte, el concepto de 
metáfora computacional sobre el cual se desarrollan permanece muy similar al que se desarrolló en sus inicios.

Las consideraciones que preocuparían a una ciencia cognitiva hoy en día (el ser humano como ser social, la relación emoción-cognición, la corporeidad de los procesos cognitivos) son apenas vistas superficialmente y con extremo cuidado en las pocas instancias que son mencionadas. La noción del ser humano como un ser social o incluso como un ser con emociones es apenas una idea que se cree puede traer consecuencias en cuanto al planteamiento de futuros esfuerzos sobre memoria humana y la metáfora computacional. Esta anuencia a no enfrentar estos retos se debe, posiblemente, a la incapacidad de dar cabida a toda esta complejidad dentro de las limitaciones de modelo o sistema en una máquina computacional, lo cual sugiere nuevamente que las ideas de la metáfora computacional, como tradicionalmente se han tratado, resultan insuficientes.

Las propuestas que más cuestionan los fundamentos de la metáfora computacional son las basadas en sistemas dinámicos donde se plantea la cognición como la instanciación de un sistema basado en procesos que se modifican en el tiempo y no en uno basado en la manipulación de estructuras simbólicas tradicionales. Para ver como se diferencian realmente estos sistemas de algunas propuestas conexionistas, las cuales mantienen un sesgo computacional de fondo, se debe profundizar en la teoría.

\section{Discusión y conclusión}

La propuesta original de la IA perdió fuerza hace ya varios años y esto se evidencia en la intensión de los autores en los planteamientos de las propuestas computacionales sobre memoria. Muchos autores hoy en día se refieren a aplicaciones de simulación y modelación donde se diferencia que la máquina no va a emular inteligencia humana, sino que va a servir como herramienta para comprender la cognición. Este acercamiento no es tan claro en las propuestas sobre arquitecturas cognitivas donde hay una voluntad de proponer teorías computacionales básicas sobre la cognición e implementarlas lo más fielmente posible (Sun, 2009). A pesar de que parece estar en su mayor parte superada la etapa de la IA fuerte y de la metáfora computacional, lo que se evidencia en las poca cantidad de propuestas que tratan el tema directamente, se puede identificar que hay sesgos de fondo más fuertes que influyen sobre el estudio de la memoria desde la perspectiva computacional y que influyen en otras disciplinas del estudio de los procesos cognitivos humanos.

Si bien la mayoría de propuestas tratan sobre herramientas de estudio de la memoria, la estructura de la máquina computacional calza muy bien con la teoría de la metáfora computacional de la mente, por lo que el sesgo proveniente de sus supuestos teóricos es casi inevitable. Esto se evidencia en el poco impacto que provocan las propuestas en computación en el área del estudio de la memoria y en los resultados pobres que se reportan como retroalimentación para otras disciplinas.

La metáfora computacional de la mente plantea que la cognición es producto de una serie de manipulaciones sobre estructuras simbólicas formales. De esta forma se pueden manipular contenidos semánticos mediante estructuras sintácticas que se modifican con mecanismos de causalidad (Horst, 2005). Esto, sin embargo, promueve que la visión de la mente se reduzca a un sistema contenido, modular e individual con una visión pobre y poco intuitiva de lo que es el ser humano, como se evidencia en la concepción que se maneja de éste, así como en la concepción de conocimiento, emociones, lenguaje y naturaleza social de la especie.

Sí existen propuestas que intentan incluir ciertos de estos aspectos como relevantes para el estudio de la memoria siempre desde la perspectiva de la metáfora computacional. J. R. Anderson incluye en 
su arquitectura Act- $\mathrm{R}$ módulos de visión y de tacto (con la mano) para una simulación de una cognición con un cuerpo. Ron Sun plantea la importancia de la interacción social en una propuesta basada en agentes y describe un sub-sistema motivacional, lo cual sugiere un componente emocional superficial (Sun, 2004), Robert Marinier y John Laird, por su parte, proponen la integración de un sistema que maneje emociones en la arquitectura cognitiva multiagente SOAR (Marinier et ál., 2004). Otras propuestas se limitan a proponer la inclusión de ciertos de estos aspectos en la realización de modelos y aplicaciones sobre la memoria (Kallinen et ál., 2004, Sas et ál., 2006). Ninguna de estas propuestas cuestiona directamente los supuestos de la metáfora computacional y lo que plantean es la idea de incluir modificaciones a propuestas de modelos de memoria existentes pero, se sospecha que se necesitan cambios más profundos en la concepción misma de la cognición y no parches o módulos extra para provocar resultados verdaderamente interesantes.

La propuesta más interesante es la basada en sistemas dinámicos que empieza por una crítica a fondo de los supuestos de la metáfora computacional y plantea modelos computacionales para simulación que trabajen sobre los principios de la teoría de sistemas dinámicos. En esta propuesta la cognición se considera como un fenómeno ligado al cuerpo, a las emociones, a las interacciones sociales y a los procesos evolutivos que se desenvuelve dentro de un contexto (van Gelder, 1998, Chartier et ál., 2008, Izquierdo, 2008). Desde esta perspectiva, el estudio de la memoria desde un enfoque computacional puede resultar interesante y permite pensar fuera de los límites tácitamente impuestos en la investigación en el campo.

A pesar de estas excepciones, el área de estudio de la memoria parece estar en un ambiente muy endogámico. Es necesario que más propuestas que estudien la memoria desde el campo de la computación se concentren en cuestionar los fundamentos base de la metáfora computacional para proponer luego teorías y modelos que hagan frente a los problemas más significativos.

\section{Agradecimientos}

A Andrés Segura Castillo por sus sugerencias y ayuda en la revisión de la bibliografía.

\section{Notas}

1 El tamiz utilizado se encuentra como anexo.

2 Uno de los principales problemas que rodean a la inteligencia artificial es que no existe un consenso sobre lo que es inteligencia. Tanto problema había con definir el término que la única forma de evaluar si una máquina era inteligente o no era realizando una conversación entre un ser humano y una máquina y, en caso de que el primero no pudiera distinguir que la máquina no era un humano se consideraría a la máquina como inteligente. Esta prueba se llamó la prueba de Turing (Turing 1950) y fue criticada fuertemente, entre otros, por John Searle con su argumento del cuarto chino (Searle 1980).

3 pág. 424.

4 págs. 21-25.

5 Para un desarrollo más ámplio sobre este tema ver Horst (2005).

6 Las fuentes escogidas se encuentran marcadas con un asterisco en las referencias bibliográficas.

7 Esto no es completamente claro en la propuesta de sistemas dinámicos que se mencionará más adelante.

\section{Referencias bibliográficas}

ACT-R Research Group. (s. f.). ACT-R. Recuperado de http://act-r.psy.cmu. edul

*Anderson, J. R. (1996). ACT: A simple theory of complex cognition. American Psychologist, 51, 355-365. 
Anderson, J. R. y Betz, J. (2002). A hybrid model of categorization. Psychonomic Bulletin \& Review, 8, 629-647.

Anderson, J. R., Bothell, D., Lebiere, C., y Matessa, M. (1998). An integrated theory of list memory. Journal of Memory and Language, 38, 341-380.

Anderson, J. R., Budiu, R. y Reder, L. M. (2001). A theory of sentence memory as part of a more general theory of memory. Journal of Memory and Language, 45, 337-367.

*Aizenbud-Reshef, N., Belinsky, E., Jacovi, M., Laufer, D. y Soroka, V. (2008). Pensieve. Augmenting human memory. CHI '08 extended abstracts on Human factors in computing systems, 3231-3236.

*Baddeley, A. D. (1986). Working memory. New York: Oxford University Press.

*Burgess, C. (2000). Theory and operational definitions in computational memory models: A response to Glenberg and Robertson. Journal of Memory and Language, 43, 402-408.

*Burgess, N. y Hitch, G. (2005). Computational models of working memory: putting long-term memory into context. Trends in Cognitive Sciences, 9(11), 535-541.

*Byrne, M. D. (1996) A computational theory of working memory. Conference companion on Human factors in computing systems: common ground, 31-32.

*Chartier, S., Renaud, P., y M. Boukadoum. (2008). A nonlinear dynamic artificial neural network model of memory. New Ideas in Psychology, 26, 252-277.

${ }^{*}$ Collins, A. M. (1973). Studies of human memory and language processing.
(Reporte). Washington, DC: National Inst. of Education (DHEW).

*Commarford, P. M. (2006). Working memory, search, and signal detection: implications for interactive voice response system menu design. (Tesis doctoral), University of Central Florida, United States, Florida.

Dawson, J. W. (2006). Goedel and the Limits of Logic. Plus Magazine, 39. Disponible en http://plus.maths.org/ issue 39/features/dawson/

*Dekel, U. (2008). Designing a prothetic memory to support software developers. Companion of the 30th international conference on Software engineering, 1011-1014.

*Denhière, G. y Lemaire, B. (2004). A computational model of children's semantic memory. In $\mathrm{K}$. Forbus, D. Gentner y T. Regier (Eds.). Proceedings of the 26 th Annual Meeting of the Cognitive Science Society (pp. 297- 302), Chicago, Estados Unidos.

*Elsweiler, D. (2007). Supporting human memory in personal information management. (Tesis doctoral). Department of Computer and Information Sciences at the University of Strathclyde. Glasgow, Esocia.

*Foltz, P. W. (1991). Models of human memory and computer information retrieval: Similar approaches to similar problems (Reporte). Estados Unidos: University of Colorado, Institute of Cognitive Science.

*Hewett, T. T. (2005). Designing with the human memory in mind. Proceedings of the 7th international conference on Human computer interaction with mobile devices \& services, 363-364. 
Horst, S. (2005). The Computational Theory of Mind. The Stanford Encyclopedia of Philosophy, Fall 2005 Edition. Disponible en http://plato.stanford. edu/archives/fall2005/entries/ computational-mind/

*Howard, M. W. (2009). Computational models of memory. In L.R. Squire (Eds.). Encyclopedia of Neuroscience, 5, (pp.771-777). Oxford: Academic Press.

*Huggett, M., Hoos, H. y Rensink, R. (2007). Cognitive principles of information management: The principles of mnemonic associative knowledge (P-MAK). Minds and Machines, 17(4), 445-485.

*Huguenard, B. R., Lerch, F. J., Junker, B. W., Patz, R. J. y Kass, R. E. (1997). Working-memory failure in phonebased interaction. ACM Transactions on Computer-Human Interaction (TOCHI), 4(2), 67-102.

*Izquierdo, E. (2008). The dynamics oflearning behaviour: A situated, embodied, and dynamical systems approach. (Tesis doctoral). Universidad de Sussex. Disponible en http://eduardoizquierdo. tumblr.com/phd

*Kallinen, K., Laarni, J., Ravaja, N., y Saari, T. (2004). Gender and habitual use of media moderate the memory performance in emotional-multimodal context. Nordic Conference on HumanComputer Interaction, 82, 397-400.

*Kawamura, T., Fukuhara, T., Takeda, H., Kono, Y. y Kidode, M. (2006). Ubiquitous memories: a memory externalization system using physical objects. Personal and Ubiquitous Computing, 11(4), 287-298.

*Leake, D. B. (1994). Towards a computer model of memory search stra- tegy learning. Proceedings of the Sixteenth Annual Conference of the Cognitive Science Society, 549-554.

*Lehman, J. F., Laird, J. y Rosenbloom, P. (2006). A gentle introduction to SOAR: 2006 update. (Reporte). Disponible en http://ai.eecs.umich. edu/soar/sitemaker/docs/misc/ GentleIntroduction-2006.pdf

*Marinier, R. P., y Laird, J. E. (2004). Toward a Comprehensive Computational Model of Emotions and Feelings. Paper presented at the Sixth International Conference on Cognitive Modeling. Pittsburgh, Pennsylvania, Estados Unidos.

McCarthy, J., Minsky, M. L., Rochester, N., y Shannon, C. E. (1955). A Proposal for the Dartmouth Summer Research Project on Artificial Intelligence. In Chrisley, R. (eds.). Artificial Intelligence: Critical Concepts, 2, (pp. 4453). Nueva York, Estados Unidos.

McCurdock, P. (2004). Machines who think: A personal inquiry into the history and prospects of artificial intelligence ( $2^{\text {a }}$ ed.). Massachusetts, Estados Unidos: A. K. Peters, Ltd.

*Norman, K. A, Detre, G. y Polyn, S. M. (2008). Computational models of episodic memory. In R. Sun (Ed.). The Cambridge HandbookofComputational Psycholog, (189-225). Cambridge University Press, Cambridge.

Newell, A. (1990). Unified Theories of Cognition. Cambridge, Massachusetts: Harvard University Press.

Newell y Simon (1959). The Simulation of Human Thought. Santa Mónica, California: Rand Corporation.

Nordlander, T. E. (2001). AI Surveying: Artificial Intelligence in Business. 
(Tesis de Maestría), Universidad de Montford, Montford, Leicester.

*O'Reilly, R. C., Braver, T. S. y Cohen, J. D. (1999). A biologically-based computational model of working memory. In A. Miyake y P. Shah (Eds.). Models of Working Memory: Mechanisms of Active Maintenance and Executive Control. (pp. 375-411), New York: Cambridge University Press.

*Rizzuto, D. S. (2002). The computational and electrophysiological foundation of item and associative memory. (Tesis doctoral). The Faculty of the Graduate School of Arts and Sciences, Brandeis University, Waltham/ Boston, Massachusetts, Estados Unidos.

*Rogers, T. T. (2008). Computational models of semantic memory. In R. Sun (Ed.). The Cambridge Handbook of Computational Psychology, 226-266. New York: Cambridge University.

Russell, S., y Norvig, P. (2009). Artificial Intelligence: A Modern Approach ( $3^{\mathrm{a}}$ ed.). Nueva Jersey, Estados Unidos: Prentice Hall.

*Sas, C., y Dix, A. (2006). Designing for collective remembering. Conference on Human Factors in Computing Systems, 1727-1730.

*Schoelles, M. J., Neth, H., Myers, C. W., y Gray, W. D. (2006). Steps towards integrated models of cognitive systems:A levels-of-analysis approach to comparing human performance to model predictions in a complex task environment. In Proceedings of the 28th Annual Conference of the Cognitive Science Society (pp. 756761) . Vancouver, British Columbia, Canadá.
*Schweer, A. y Hinze, A. (2007). Challenges in interface and interaction design for context-aware augmented memory systems. Proceedings of the 7th ACM SIGCHI New Zealand chapter's international conference on Computerhuman interaction: design centered HCI, 55-58.

Searle, J. R. (1980). Minds, Brains, and Programs. The Behavioral and Brain Sciences, 3, 417-457.

*Shastri, L. (2001). A computational model of episodic memory formation in the hippocampal system. Neurocomputing, Volúmenes 38-40: 889-897.

*Steyvers, S., Griffiths, T. L., y Dennis, S. (2006). Probabilistic inference in human semantic memory. TRENDS in Cognitive Sciences, 10(7).

*Sun, R. (2004). The CLARION Cognitive Architecture: Extending Cognitive Modeling to Social Simulation. In Sun, R. (eds). Cognition and MultiAgent Interaction, (pp. 79-103). Nueva York, Estados Unidos: Cambridge University Press.

Sun, R. (2009). Theoretical status of computational cognitive modeling. Cognitive Systems Research, 10(2), 124-140.

Turing, A. (1950). Computing Machinery and Intelligence. Mind, 49, 433-460.

*Van den Hoben, E., Eggen, B. (2007). Informing augmented memory system design through autobiographical memory theory. Personal and Ubiquitous Computing, 12(6), 433-443.

*Van Gelder, T. (1998). The Dynamical Hypothesis in Cognitive Science. The 
Behavior and Brain Sciences, 21, Von Eckardt, B. (1993). What is cognitive 615-665.

*Vemuri, S., Schmandt, C. y Bender, W. science? Cambridge, Massachusetts: The MIT Press. (2006). ¡Remember: A personal, longterm memory prosthesis. Proceedings of the 3rd ACM workshop on Continuous archival and retrival of personal experences, 65-74.

*West, D., Quigley, A. y Kay, J. (2007). MEMENTO: a digital-physical scrapbook for memory sharing. Personal and Ubiquitous Computing, 11(4), 313-328. 


\section{Anexo 1}

\section{Documento utilizado para la revisión de propuestas}

\section{TAMIZ}

\section{Propuesta}

Se revisarán las propuestas sobre la memoria humana de acuerdo con el siguiente tamiz que toma en cuenta una serie de instancias epistémicas y procedimientos de análisis (validez interna y externa de la teoría, coherencia teórica, plausibilidad, noción de proceso, conectividad inter e intra-teórica).

Las posiciones teóricas desde la cuales se hará la revisión son las siguientes:

1. Emociones:

2. Lenguaje:

3. Arquitecturas cognicionales:

4. Naturaleza social de la especie:

\section{ASPECTOS GENERALES}

Nombre de la propuesta:

\section{Origen de propuesta}

Psicología

$\square \quad$ Neurociencias

$\square \quad$ Informática /Inteligencia artificial

$\square \quad$ Ciencias Cognitivas

Filosofía

\section{Otros}

- Detalle de aspectos más específicos del origen tales como enfoque, fecha de origen, autor/autores:

- ¿Qué problema busca resolver la propuesta?: 


\section{Descripción de la propuesta}

- Detalle en que consiste la propuesta, cuáles son sus fundamentos teóricos de fondo, y cuáles las principales líneas de desarrollo.

- ¿El contenido de la propuesta es sobre memoria propiamente o se trata de una propuesta que entre sus contenidos se encuentra el tema de memoria?:

- Detalle el método como se intenta resolver el problema (teóricamente, empíricamente, y la estructura de estos formatos)

- La propuesta se autodefine como:

$\begin{array}{ll}\square & \text { Hipótesis } \\ \square & \text { Teoría } \\ \square & \text { Modelo } \\ \square & \text { Otro: }\end{array}$

- La propuesta luego del tamiz es considerable como:

$\begin{array}{ll}\square & \text { Hipótesis } \\ \square & \text { Teoría } \\ \square & \text { Modelo } \\ \square & \text { Otro: }\end{array}$

- ¿La propuesta es molar o molecular?

\section{Metodología de la propuesta}

- Nivel de la propuesta: exploratoria, descriptiva, teórica-X, explicativa, otros niveles.

- Evaluación de su validez: aporta evidencias sobre la propuesta, son contundentes estas evidencias, trabaja con dominios de validez, usa validez semántica, validez por criterios, validez interna y externa, criterios de triangulación, validez conceptual, de variables o estadística.

- Evaluación de la fiabilidad: a nivel muestral, de universo, técnicas de recolección de la información, instrumental.

- Evaluación de la implementación metodológica: hay estandarización metodológica, existen marcos de interpretación claros y concisos, hay claridad epistémica en los conceptos de fondo.

- Plausibilidad: es realista la propuesta, tiene implantación histórica-social, cultural, diferencial.

- ¿La propuesta es falseable?

- ¿Qué tan potente y evidente es el manejo del lenguaje, las emociones, la naturaleza social de nuestra especie, las arquitecturas cognicionales, en esta propuesta? 


\section{Nociones Epistémicas de fondo}

- ¿Cuál es la noción de ser humano que tiene la propuesta?

- ¿Cómo maneja el concepto de información?

- ¿Cómo entiende el concepto de conocimiento?

- ¿Cómo relaciona la propuesta con otras propuestas?

- Podemos clasificar la propuesta de acuerdo a su alcance en la explicación del fenómeno de la memoria humana como:

$\square$ Corto alcance

$\square$ Mediano alcance

$\square$ Alto alcance

- ¿La propuesta axiomatiza?, ¿categoriza resultados?

- ¿La propuesta es modelable computacionalmente?

- ¿Qué nivel de compatibilidad nuclear (Lakatos) tiene la propuesta con otras propuesta?

- ¿Qué aspectos ideológicos: políticos, económicos, religiosos, científicos hay detrás de esta propuesta?

\section{Impacto y alcances}

- ¿Afecta la propuesta otras propuestas de la memoria humana? De ser afirmativo, ¿cómo?

- ¿El lenguaje de la propuesta es asimilable por enfoque a otras propuestas?

- ¿Modifica la propuesta, de alguna manera, el horizonte de la investigación en memoria humana?

- ¿Qué aportes da la teoría a aspectos como la educación, la salud, la economía, etc.?

- ¿Qué tan pertinente es esta propuesta para nuestro contexto nacional y académico? 